caused ballismus movements in this case. In patients who present with short duration monoballismus, evaluation for subthalamic nuclei function, seizure disorders and other origins of ballismus are warranted.

FUNDING ACKNOWLEDGEMENTS: Smell \& Taste Treatment and Research Foundation

127

\section{Cardiovascular Safety Assessment of Deutetrabenazine in Healthy Volunteers and Implications for Patients With Huntington Disease or Tardive Dyskinesia}

Donna S. Cox, PhD'; Micha Levi, PhD'; Laura Rabinovich-Guilatt ${ }^{\prime}$; David Truong, PharmD, $\mathrm{MS}^{\prime}$; and David Stamler, $M D^{\prime}$

${ }^{1}$ Teva Pharmaceutical Industries, Frazer, Pennsylvania, USA

ABSTRACT: Introduction: Deutetrabenazine is approved for treating Huntington disease (HD) chorea and is being evaluated for tardive dyskinesia (TD).

OBJECTIVE: To assess the effect of deutetrabenazine on cardiac repolarization.

METHODS: A QT interval study was performed to evaluate effects of deutetrabenazine 12 and $24 \mathrm{mg}$ on cardiac repolarization, as assessed by time-matched change from baseline, placebo-adjusted, in Fridericia-corrected QT interval $(\Delta \Delta \mathrm{QTcF})$. Moxifloxacin $(400 \mathrm{mg})$ and tetrabenazine $(50 \mathrm{mg})$ were the positive control and comparator, respectively. An exposure-response analysis was developed from this study to predict maximal effects on QTcF at maximum recommended dosing based on CYP2D6 status, an approach consistent with regulatory guidance at predicting QT interval effects.

RESULTS: Maximal $\Delta \Delta \mathrm{QTcF}$ between the least-squares mean (90\% two-sided confidence interval) of deutetrabenazine 12 and $24 \mathrm{mg}$ ( $\mathrm{n}=45$ in each group) were 2.8 $(0.7-4.8) \mathrm{ms}$ and $4.5(2.4-6.5) \mathrm{ms}$, respectively. The $\Delta \Delta \mathrm{QTcF}$ increase with tetrabenazine $(\mathrm{n}=45)$ was 7.6 (5.6-9.5) ms. Assay sensitivity was verified with moxifloxacin $(\mathrm{n}=47)$, which produced a maximal effect on $\Delta \Delta$ QTcF of 14.0 (11.9-16.0) ms. A linear model was developed that described a correlation between plasma concentrations from pivotal HD andTD trials $(n=101)$ and QT interval prolongation. Using that model and the individual predicted Cmax for HD and TD patients, the placebo-adjusted change from baseline inQTcF for deutetrabenazine at maximal recommended daily doses was found to be $5.4(2.5-9.5) \mathrm{ms}$.
CONCLUSIONS: Patients receiving the maximal recommended doses of deutetrabenazine are predicted to have a QTcF increase below the level of regulatory concern.

Presented at: Psych Congress; September 16-19, 2017; New Orleans, Louisiana, USA.

FUNDING ACKNOWLEDGEMENTS: This study was funded by Teva Pharmaceutical Industries, Petach Tikva, Israel

128

Effect of DR/ER-MPH on Early Morning and Late Afternoon/Evening Functioning in Children With ADHD: Analysis of PREMB-R Items From a Phase 3 Trial

Steven R. Pliszka, MD'; Valerie K. Arnold, $M D^{2}$; Andrea Marraffino, PhD ${ }^{3}$; Norberto J. DeSousa, $M A^{4}$; Bev Incledon, $P h D^{4} ; F$. Randy Sallee, $M D, P h D^{4}$; Timothy E. Wilens, $M D^{5}$; and Jeffrey $H$. Newcorn, $M D^{6}$

${ }^{1}$ The University of Texas Health Science Center at San Antonio, San Antonio, Texas, USA

${ }^{2}$ University of Tennessee Health Science Center, Memphis, Tennessee, USA

${ }^{3}$ Meridien Research, Inc., Maitland, Florida, USA

${ }^{4}$ Ironshore Pharmaceuticals \& Development, Inc., Camana Bay, Grand Cayman, Cayman Islands

${ }^{5}$ Massachusetts General Hospital, Boston,

Massachusetts, USA

${ }^{6}$ Mount Sinai Medical Center, New York, New York, USA; On behalf of the HLD200-108 Study Group

ABSTRACT: Objective: In a phase 3 trial of children with ADHD, DR/ER-MPH (formerly HLD200), a delayedrelease and extended-release methylphenidate, improved ADHD symptoms and reduced at-home early morning and late afternoon/evening functional impairments versus placebo, as measured by the validated Parent Rating of Evening andMorning Behaviors-Revised, Morning (PREMB-R AM) and Evening (PREMB-R PM) subscales. This post hoc analysis evaluated the effect of DR/ER-MPH versus placebo onindividual PREMB-R $\mathrm{AM} / \mathrm{PM}$ item scores.

METHOD: Data were analyzed from a pivotal, randomized, double-blind, multicenter, placebo-controlled, parallelgroup, phase 3 trial of DR/ER-MPH in children (6-12 years) withADHD (NCT02520388). Using the 3-item PREMB-R AM and 8-item PREMB-R PM, both key secondary endpoints, investigators evaluated early morning and lateafternoon/evening functional impairment by scoring each item on a severity scale from 0 (none) to 3 (a lot). For post hoc analyses, treatment comparisons between DR/ER-MPH and placebo at endpoint were determined by using least squares mean changes from 
baseline on individual PREMB-R AM/PM items score derived from an analysis ofcovariance (ANCOVA) model with treatment as the main effect, and study center and baseline score as covariates.

RESULTS: Of 163 children enrolled across 22 sites, 161 were included in the intent-to-treat population (DR/ER$\mathrm{MPH}, \mathrm{n}=81$; placebo, $\mathrm{n}=80$ ) and 138 completed the study. The mean DR/ER-MPH dose achieved after 3 weeks of treatment was $68.1 \mathrm{mg}$. Following 3 weeks of treatment, DR/ER-MPH significantly reduced mean individual item scores from baseline versus placebo on all PREMB-R AM items (all P $\leq 0.002$; "getting out of bed", "getting ready", and "arguing or struggling in the morning"). Additionally, DR/ER-MPH significantly reduced mean individual item scores from baseline on 5 out of 8 PREMB-R PM items $(\mathrm{P}<0.01$ in 2 items ["sitting through dinner" and "playing quietly"] and $\mathrm{P}<0.05$ in 3 items ["inattentive/distractible", "transitioning between activities", and "settling down/getting ready for bed"]). There was a trend towards a reduction on 2 other items of the PREMB-R PM $(\mathrm{P}<0.09)$. Distributions of the ratings for each item will be presented. No serious TEAEs were reported; TEAEs were consistent withmethylphenidate.

CONCLUSIONS: Post hoc analyses revealed that DR/ERMPH significantly reduced all PREMB-R AM item scores, including "getting out of bed", and many PREMB-R PM items, including "getting ready for bed" in children with ADHD. These findings are worth further exploration.

FUNDING ACKNOWLEDGEMENTS: Ironshore Pharmaceuticals \& Development, Inc.

\section{9}

\section{Effect of DR/ER-MPH on Caregiver-Reported ADHD Symptom Improvement in Children With ADHD and Caregiver Strain: Results From a Phase 3 Trial}

Steven R. Pliszka, $M D^{\prime}$; Valerie K. Arnold, $M D^{2}$; Andrea Marraffino, $\mathrm{PhD}^{3}$; Norberto J. DeSousa, $M A^{4}$; Bev Incledon, $P h D^{4} ; F$. Randy Sallee, $M D, P h D^{4}$; Timothy E. Wilens, $M D^{5}$; and Jeffrey H. Newcorn, $M D^{6}$

${ }^{1}$ The University of Texas Health Science Center at San Antonio, San Antonio, Texas, USA

${ }^{2}$ University of Tennessee Health Science Center, Memphis, Tennessee, USA

${ }^{3}$ Meridien Research, Inc., Maitland, Florida, USA

${ }^{4}$ Ironshore Pharmaceuticals \& Development, Inc., Camana Bay, Grand Cayman, Cayman Islands

${ }^{5}$ Massachusetts General Hospital, Boston,

Massachusetts, USA
${ }^{6}$ Mount Sinai Medical Center, New York, New York, USA; On behalf of the HLD200-108 Study Group

ABSTRACT: Objective: Evening-dosed DR/ER-MPH (formerly HLD200), a delayed-release and extended-release methylphenidate, was designed to provide efficacy upon awakening and through the evening. The objective was to evaluate whether treatment with DR/ER-MPH in children with attention-deficit/hyperactivity disorder (ADHD): (1) improves caregiver-rated ADHD symptoms, and (2) reduces caregiver strain, versus placebo.

METHOD: Caregiver-rated ADHD symptoms (Conners' Global Index-Parent [CGI-P]) and caregiver strain (Caregiver Strain Questionnaire [CGSQ]) were assessed as secondary endpoints following 3 weeks of treatment in a randomized, double-blind, multicenter, placebo-controlled, parallel-group, phase 3 trial of DR/ER-MPH in children (6-12 years) with ADHD (NCT02520388). Using the 10-item CGI-P, parents rated their child's ADHD symptoms on a 4 -point scale $(0=$ never/seldom; $3=$ very often/frequently). Caregivers also rated the impact of caring for a child with emotional and behavioral challenges on the 21-item CGSQ (5-point scale: $1=$ not at all; $5=$ very much). A reduction on individual item and total scores for both measures indicated an improvement.

RESULTS: Of 163 children enrolled across 22 sites, 161 were included in the intent-to-treat population (DR/ER-MPH, $\mathrm{n}=81$; placebo, $\mathrm{n}=80$ ) and 138 completed the study. The mean DR/ER-MPH dose after 3 weeks of treatment was $68.1 \mathrm{mg}$. Mean CGI-P scores at baseline and CGSQ scores at screening (ie, before washout of prior ADHD therapy) were comparable for both DR/ER-MPH (CGI-P: 22.8, CGSQ: 54.5) and placebo (CGI-P: 21.8; CGSQ: 54.9) groups. After 3 weeks of treatment, caregivers of children onDR/ER-MPH reported significant reductions in CGI-P scores versus those on placebo (least-squares [LS] mean: 12.3 vs $17.4 ; \mathrm{P}<0.001$ ). Additionally, there was a significant reduction in CGSQ scores after 3 weeks of treatment with DR/ER-MPH versus placebo (LS mean: 41.2 vs 49.1; $\mathrm{P}<0.001)$. Post hoc analyses on the effect of DR/ER-MPHversus placebo on individual items of CGI-P and CGSQ, and the two subscales of CGI-P will be presented. No serious TEAEs were reported and all TEAEs were consistent with those of MPH.

CONCLUSIONS: Caregivers reported significant improvements in their child's ADHD symptoms and these improvements coincided with reductions in caregiver strain after 3 weeks of treatment on evening-dosed DR/ER-MPH versus placebo.

FUNDING ACKNOWLEDGEMENTS: Ironshore Pharmaceuticals \& Development, Inc. 